\title{
SITUACIÓN DEL NEUROMARKETING EN ESPAÑA
}

\section{Celia Andreu-Sánchez, Aroa Contreras-Gracia y Miguel-Ángel Martín-Pascual}

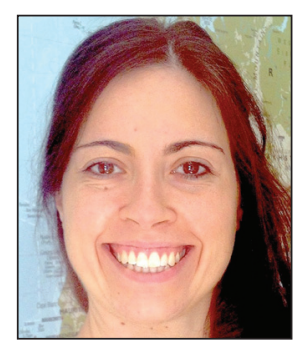

Celia Andreu-Sánchez es doctora en comunicación y máster en neurociencias y psicobiología del comportamiento. Es directora del grupo de investigación Neuro-Com, dedicado a la investigación de neurociencias en ciencias de la comunicación, en la Universitat Autònoma de Barcelona (UAB), miembro de la División de Neurociencias de la Universidad Pablo Olavide de Sevilla, profesora de comunicación audiovisual, publicidad y relaciones públicas y periodismo en la UAB y en la Universitat de Girona. Es gestora de comunicación online y desarrolladora de iOS para Apple Inc. http://orcid.org/0000-0001-9845-8957

Universitat Autònoma de Barcelona, Facultat de Comunicació. Edif. I, Dept. de Comunicació Audiovisual i Publicitat Campus Bellaterra 08193 Cerdanyola del Vallès, Barcelona, España celia.andreu@uab.cat

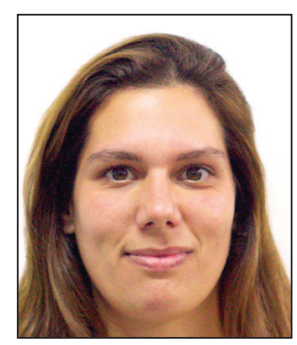

Aroa Contreras-Gracia es graduada en publicidad y RRPP por la Universitat de Girona (2013) tras una trayectoria académica formativa en distintos centros universitarios españoles: Universitat de Girona, Universidad San Jorge y Universidad de Sevilla. Ha realizado prácticas en una empresa española puntera en el desarrollo e investigación en tecnología $\mathrm{BCl}$ (brain computer interfaces). Apasionada por el mundo de la innovación y sobre todo por las neurociencias aplicadas a la comunicación, concretamente a la publicidad.

http://orcid.org/0000-0003-2920-3605

Universitat de Girona, Facultat de Turisme Edif. Sant Domènec, PI. Ferrater Mora, 1. 17071 Girona, España aroacontrerasgracia@gmail.com

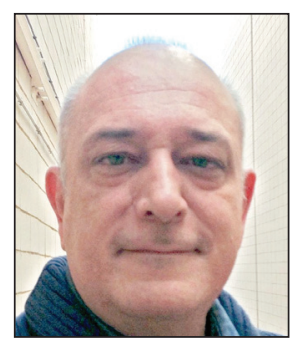

Miguel-Ángel Martín-Pascual es profesor de comunicación audiovisual y publicidad en la Universitat Autònoma de Barcelona y director de formación en el Instituto de Radiotelevisión Española en Barcelona. Es realizador de televisión en Televisión Española, desarrollador de iOS para Apple Inc y miembro de la División de Neurociencias de la Universidad Pablo Olavide de Sevilla. Es máster en neurociencias y psicobiología del comportamiento.

http://orcid.org/0000-0002-5610-5691

Universitat Autònoma de Barcelona, Facultat de Comunicació. Edif. I, Dept. de Comunicació Audiovisual i Publicitat Campus Bellaterra 08193 Cerdanyola del Vallès, Barcelona, España miguelangel.martin@uab.cat

\section{Resumen}

El neuromarketing existe desde hace poco más de una década, y está despertando el interés de la investigación científica. En estos años se ha definido el marco teórico, las técnicas y los métodos de investigación. La ausencia de una legislación que regule cualquier experimento en neuromarketing y el hermetismo del sector privado debido a sus clientes hace difícil conocer el uso de esta disciplina por las marcas. 16 de los 75 centros universitarios españoles realizan investigación en neuromarketing, pero no tiene repercusión en los índices de impacto internacionales. Por su parte, una quinta parte del sector publicitario profesional español muestra interés real en neuromarketing, bien teniendo un departamento propio dedicado al mismo, o bien ofreciendo los servicios pertinentes. La ética, presentada tradicionalmente como un impedimento para el desarrollo del neuromarketing, no debe suponer un problema para la evolución de este campo de conocimiento.

\section{Palabras clave}

Neuromarketing, Marketing, Comunicación, Publicidad, Agencias, Investigación, Neurociencia, Neuroética, Ética.

\section{Title: Situation of neuromarketing in Spain}

\section{Abstract}

Neuromarketing has existed for just over a decade, and its theoretical framework, techniques and research methods have been defined during these years. The absence of legislation regulating neuromarketing experiments and the secrecy of the 
private sector due to its client agreements, it is difficult to know how brands use this discipline. Currently, the scientific sector in Spain has great interest in neuromarketing. Sixteen of Spain's 75 universities (21\%) have a research group, institute or center that studies neuromarketing, but this activity has had little international impact. Meanwhile, a fifth of the Spanish professional commercial sector shows real interest in neuromarketing, either having a separate department dedicated to it or offering this service to others. Ethics, traditionally presented as an impediment to the development of neuromarketing, should not pose a problem for the development of this field of knowledge.

\section{Keywords}

Neuromarketing, Marketing, Communication, Advertising, Agencies, Research, Neuroscience, Neuroethics, Ethics.

Andreu-Sánchez, Celia; Contreras-Gracia, Aroa; Martín-Pascual, Miguel-Ángel (2014). "Situación del neuromarketing en España". El profesional de la información, marzo-abril, v. 23, n. 2, pp. 151-157.

http://dx.doi.org/10.3145/epi.2014.mar.07

\section{El neuromarketing}

El neuromarketing es una reciente área de conocimiento en la que, utilizando técnicas y conocimientos de neurociencia, se investiga cómo se puede mejorar el marketing, la comunicación publicitaria y, en definitiva, el aumento de ventas de un producto o marca determinados. Son varias las definiciones que podríamos tener en cuenta para crear un marco teórico (Smidts, 2002; Braidot, 2007; Lee et al., 2007; Humbert; Kenning, 2008; Randall, 2009; Pradeep, 2010). Todas coinciden en que el neuromarketing estudia cómo se comporta el cerebro del consumidor, utilizando técnicas y métodos de investigación neurocientífica con el objetivo de mejorar las estrategias de marketing. Se encuentra así próximo a la investigación de mercados.

En los últimos años la producción científica sobre neurociencia ha aumentado considerablemente. El número de publicaciones en las revistas científicas de mayor prestigio se ha duplicado en una década. Mientras que en los 90 se publicaron 1.149 artículos científicos de neurociencia en revistas de prestigio internacional, durante la primera década del siglo XXI, se publicaron 2.916 (Andreu-Sánchez, MartínPascual, 2014). Además la investigación en neurociencia se ha expandido a áreas y disciplinas ajenas a ella hasta la fecha y la encontramos vinculada a la comunicación, economía, derecho penal o religión, entre otras. Esto ha provocado la creación del concepto neurocultura para hacer referencia a las ramas científicas que combinan la neurociencia con otros campos de estudio (Javor et al., 2013). Una de estas expansiones ha sido la neurociencia aplicada al consumidor,

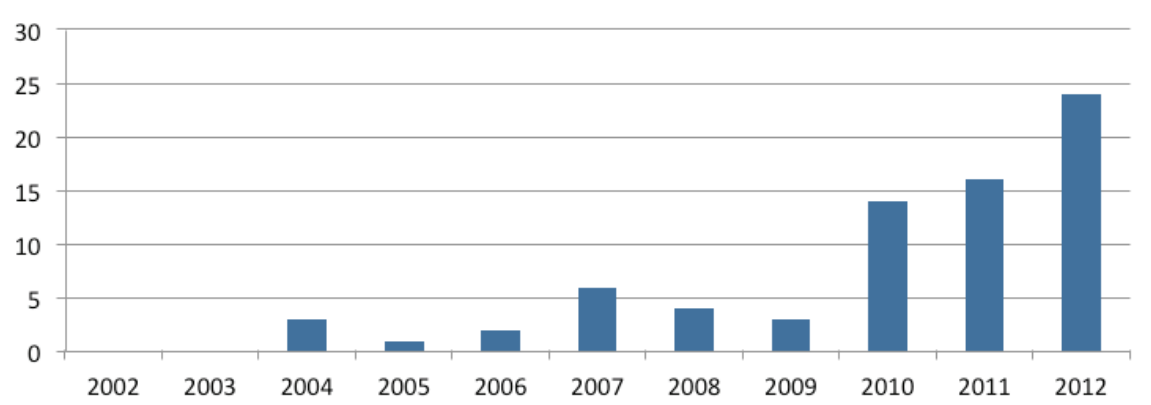

Figura 1. Artículos científicos publicados sobre neuromarketing en el siglo XXI. Fuente: Web of knowledge, Thomson Reuters. englobada en muchas ocasiones bajo el término de neuromarketing (Khushaba et al., 2013; Venkatram et al., 2011; Plassmann, 2012; Wilson et al., 2008; Weisberg et al., 2009; Ariely, 2010; Touhami et al., 2011).

Si atendemos a la base de datos Web of knowledge de Thomson Reuters, encontramos un claro aumento de las publicaciones en neuromarketing en los últimos años (figura 1). Desde que Smidts (2002) acuñara el término hasta la actualidad, podemos ver que el crecimiento ha sido importante.

La publicación en 2004 de un artículo en la revista Neuron sobre la respuesta de 67 sujetos analizada mediante resonancia magnética funcional, frente a las marcas Coca-Cola y Pepsi, supuso la entrada de los estudios de neuromarketing en la investigación científica de primera línea (MacClure et al., 2004).

La tradicional segmentación de la población realizada hasta ahora en estudios de marketing entre características demográficas, edad, geografía, nivel económico o gustos, deja de ser relevante en la investigación en neuromarketing. Ahora interesan los procesos cognitivos y perceptivos de los clientes. Las principales técnicas que se utilizan para averiguar estos procesos son: la electroencefalografía (EEG), la magnetoencefalografía (MEG), la tomografía por emisión de positrones (PET) y la resonancia magnética funcional (fMRI). Se trata de técnicas complejas que precisan de expertos específicos de cada una y un alto periodo de adquisición de datos, comparado con los métodos tradicionales de investigación en publicidad (Plassmann et al., 2007). Por otro lado, pese a ser una técnica más propia de la psicología de la percepción que de la neurociencia, entre las técnicas propias del neuromarketing se suele incluir la investigación con eye tracking (seguimiento del movimiento de los ojos frente a la pantalla) que suele ir acompañada de estudios con EEG (Khushaba et al., 2013). Se han hecho interesantes avances técnicos en el uso de esta combinación (Plöchl et al., 2012). 
Todas estas técnicas se presentan como herramientas metodológicas nuevas para observar procesos cerebrales sin necesidad de preguntar a los consumidores directamente por sus pensamientos, memorias, evaluaciones o estrategias de toma de decisión (Plassmann et al., 2012). También ofrecen una posible mejora de la eficiencia de estrategias de marketing (Venkatraman et al., 2012). Por ello el neuromarketing no suele entenderse como un nuevo método de investigación de mercados para la creación de tácticas de marketing y publicidad, sino como una opción de obtener información complementaria sobre los procesos de elección y los tipos de consumidores (Venkatraman et al., 2012). Se ha mostrado también como una fuente de generación de ideas capaces de complementar a las teorías tradicionales de la psicología, el marketing y la economía (Plassmann et al., 2012).

\section{El neuromarketing está en observación académica y profesional hasta que se decida si aporta pruebas sólidas y sus- ceptibles de ser aplicadas a la profesión del marketing}

El neuromarketing está en fase de desarrollo hasta que la comunidad científica y profesional se pronuncie claramente sobre si aporta pruebas sólidas y susceptibles de ser aplicadas a la profesión del marketing. Por otra parte el neuromarketing es una realidad que podemos comprobar por ejemplo en la inversión en investigación de instituciones como el Marketing Science Institute y el Institute for the Stu$d y$ of Business Markets, o en que cada vez se incluye más como tema de interés en revistas académicas de publicidad y marketing (Lee et al., 2007).

Por otro lado, su aparición, además de crear interés, trae consigo un problema que se plantea en muchos campos de investigación científica: la ética. A este respecto ha recibido variadas críticas (Ariely, 2010). Una de las más recurrentes es la referente a la implicación en condicionar, manipular o limitar la libertad de elección de los consumidores. El origen de la neuroética lo encontramos en la primera mitad del siglo XX. La importancia internacional dada a la ética en la investigación neurocientífica queda ya reflejada en 1947 en la reunión fundacional de la Federation of EEG and Clinical Neurophisiology que tuvo lugar en Londres. Asistieron investigadores internacionales en electroencefalografía (EEG) con el fin de regular las investigaciones con esta técnica. En 1961 se creó la International Brain Research Organization, patrocinada por la Unesco, donde tuvo su origen el concepto de neuroética en su acepción actual. En la actualidad, la International Neuroethics Society se encarga de gestionar y aconsejar la regulación de la investigación neurocientífica con implicaciones sociales, legales éticas y políticas. En la investigación clásica en marketing no se plantean académicamente problemas éticos con el uso de la tecnología para intereses comerciales (Eaton; Illes, 2007), sin embargo el uso de una técnica que analiza el comportamiento del cerebro para su aplicación en la venta comercial, sí provoca problemas éticos. Por un lado se manifiesta la posible desprotección de cualquier grupo que pueda ser explotado por el uso del neuromarketing y, por otro lado, existe una preocupación por la autonomía del consumidor (Murphy et al., 2008). En general suele manifestarse una gran inquietud por el hecho de que los publicistas tengan acceso a sofisticadas herramientas capaces de comprender e incluso dirigir el comportamiento del consumidor con el fin último de la venta de un producto.

En España hay 32 organismos que forman la Red de Comités de Ética de Universidades y Organismos Públicos de Investigación $(R C E)$. Además, la mayoría de hospitales universitarios tienen sus propios comités de ética clínica, que también trabajan coordinadamente con las universidades. Según la Comisión Ética de Experimentación Animal y Humana (Ceeah) de la Universitat Autònoma de Barcelona (UAB), con casi treinta años de experiencia en asesoramiento ético de investigación con humanos y animales, toda investigación en neuromarketing precisa un aval ético al mismo nivel que cualquier otra investigación con humanos, si desea tener el reconocimiento de un comité ético. No existe una normativa propia exclusiva para neuromarketing, sino que la regulación en sus investigaciones se realiza según la normativa de cada comité, asociados a través de la $R C E$, y que se basan en la legislación propia de los comités de bioética de Europa, de España y de algunas comunidades autónomas. Según Josep Santaló, presidente del Ceeah, las ciencias sociales presentan un aumento de consultas a la comisión en los últimos años. Algunas áreas de investigación que tradicionalmente no realizaban consultas de ética en investigación, como son las citadas ciencias sociales, la antropología, la pedagogía o la comunicación, están mostrando un aumento de solicitudes para la aprobación de protocolos (Santaló, 2013).

Hemos trabajado con tres grupos: investigadores en neuromarketing, agencias de publicidad y empresas de investigación de mercados

Según Santaló (2013), los apriorismos no pueden invalidar el objeto de ninguna investigación. Así frente al caso de la ética en investigación de neuromarketing, el Ceeah afirma que no debe existir problema por parte de ningún comité de ética de investigación, siempre y cuando se cumplan las normas de consentimiento informado y se respeten los derechos de los sujetos de investigación. Toda investigación en neuromarketing debe cumplir con la ética propia de cualquier protocolo de investigación. Hecho esto, no existe ningún planteamiento ético diferente en una investigación propia del neuromarketing frente a cualquier otro tipo de investigación con humanos. Por otro lado desde el Ceeah afirman que, debido al uso de avanzadas técnicas de investigación neurocientífica y ante el posible diagnóstico de patologías o anomalías de los individuos participantes en la investigación, éstos tienen derecho a no saber y así debe constar en el consentimiento informado previo a la investigación (Santaló, 2013).

Ante este panorama general, el objetivo de esta investigación ha sido conocer la situación del neuromarketing en España, tanto en el ámbito científico como en el profesional. 


\section{Metodología de la investigación}

Para alcanzar el objetivo de este estudio, hemos trabajado con tres grupos de interés: investigadores en neuromarketing, agencias de publicidad y empresas de investigación de mercados, encuestándolos en los meses de abril y mayo de 2013.

Para estudiar la situación de la investigación de neuromarketing en España hemos realizado encuestas en las 75 universidades españolas. A partir de sus webs hemos buscado los grupos de investigación -grupos, institutos, centros, spinoffs y oficinas de transferencia de resultados de investigación (otris)- que mostraban alguna línea de trabajo vinculada a neuromarketing (neurociencia, publicidad, marketing, comunicación). Se han encontrado 112 áreas o grupos susceptibles de tener alguna línea de investigación relacionada, formadas por 81 grupos de investigación, 5 institutos, 4 centros especializados, 2 spin-offs y 20 otris. Hemos contactado con todas vía teléfono y correo electrónico planteándoles mediante encuesta si investigaban en alguna línea de neuromarketing. A continuación hemos distribuido las respuestas en base a la universidad a la que pertenecían para poder obtener resultados coherentes en esta línea.

\section{Una cuarta parte de las universidades españolas realiza investigación sobre neuromarketing}

Para conocer la situación del neuromarketing en el entorno profesional, nos interesaba saber si se está ofreciendo como servicio adicional por parte de las agencias de publicidad tradicionales. Para ello hemos realizado un muestreo de juicio (o de selección intencional) para obtener datos sobre la población. Hemos llevado a cabo la investigación estudiando las 31 agencias que forman la Asociación Española de Agencias de Comunicación Publicitaria (Aeacp), fundada en 1977 , cuyos miembros facturan el $85 \%$ de la inversión en publicidad gestionada a través de agencias en España (Aeacp, 2013).

http://www.agenciasaeacp.es/agencias/agencias_asociadas.asp

Considerando esta muestra como representativa, hemos contactado telefónicamente o mediante correo electrónico y les hemos planteado si la agencia cuenta con un departamento de neuromarketing o si ofrecen este servicio a sus clientes. A excepción de 2 agencias que no han dado respuesta, hemos obtenido satisfactoriamente los datos de todas las demás. A las que dan una respuesta positiva y tra-
Tabla 1. Distribución de los grupos de investigación en neuromarketing por comunidades autónomas españolas.

\begin{tabular}{|c|c|c|c|c|}
\hline & Sí & No & Relacionada & Sin respuesta \\
\hline Andalucía & 4 & 6 & 0 & 0 \\
\hline Aragón & 1 & 1 & 0 & 0 \\
\hline Asturias & 0 & 0 & 1 & 0 \\
\hline Canarias & 0 & 2 & 0 & 0 \\
\hline Cantabria & 0 & 2 & 0 & 0 \\
\hline Castilla La Mancha & 1 & 0 & 0 & 0 \\
\hline Castilla y León & 0 & 7 & 0 & 1 \\
\hline Catalunya & 1 & 7 & 2 & 2 \\
\hline Comunidad Valenciana & 2 & 3 & 0 & 2 \\
\hline Extremadura & 0 & 1 & 0 & 0 \\
\hline Galicia & 1 & 2 & 0 & 0 \\
\hline Islas Baleares & 0 & 1 & 0 & 0 \\
\hline La Rioja & 1 & 1 & 0 & 0 \\
\hline Madrid & 3 & 11 & 0 & 1 \\
\hline Murcia & 2 & 1 & 0 & 0 \\
\hline Navarra & 0 & 2 & 0 & 0 \\
\hline País Vasco & 0 & 3 & 0 & 0 \\
\hline
\end{tabular}

bajan con neuromarketing, se les plantea un cuestionario compuesto por 8 preguntas con el objetivo de conocer:

- modelo de presencia de este servicio;

- formación de los responsables del mismo;

- modelos de subcontratación si los hubiera;

- protocolos de ofrecimiento a sus clientes;

- tipos de clientes que lo solicitan;

- antigüedad del servicio en su cartera;

- número de estudios realizados en el último año; y

- \% de facturación que implica.

Para averiguar la situación del neuromarketing en las empresas españolas de investigación de mercados y así tener una visión más completa en el ámbito profesional en España, hemos llevado a cabo de nuevo un muestreo de juicio (o de selección intencional). Seleccionamos trabajar con la Asociación Nacional de Empresas de Investigación de Mercados y Opinión Pública (Aneimo) que, fundada en 1978, aglutina las 16 agencias líderes del sector. Actualmente cuenta con un volumen de negocio de las empresas asociadas que supone cerca de 300 millones de euros y en ellas trabajan más de 5.000 empleados (Aneimo, 2013).

http://www.aneimo.com/asociados.php

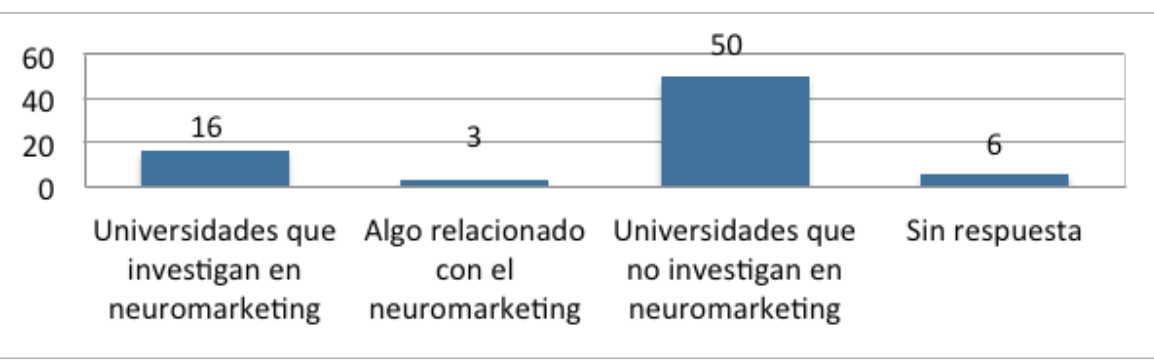

Figura 2. Universidades españolas que investigan en neuromarketing.
Los investigadores consideraron esta muestra como representativa. El método de investigación con Aneimo es idéntico al indicado antes: contacto telefónico o por correo electrónico con las empresas asociadas para averiguar si cuentan con un departamento especializado. A las empresas con respuesta afirmativa, les realizamos un segundo cuestionario 
compuesto por las mismas preguntas planteadas a las agencias de Aeacp sobre el modelo de neuromarketing en sus empresas.

\section{Resultados}

Los resultados obtenidos sobre la investigación científica indican que el $21 \%$ de las 75 universidades españolas cuenta con algún grupo,

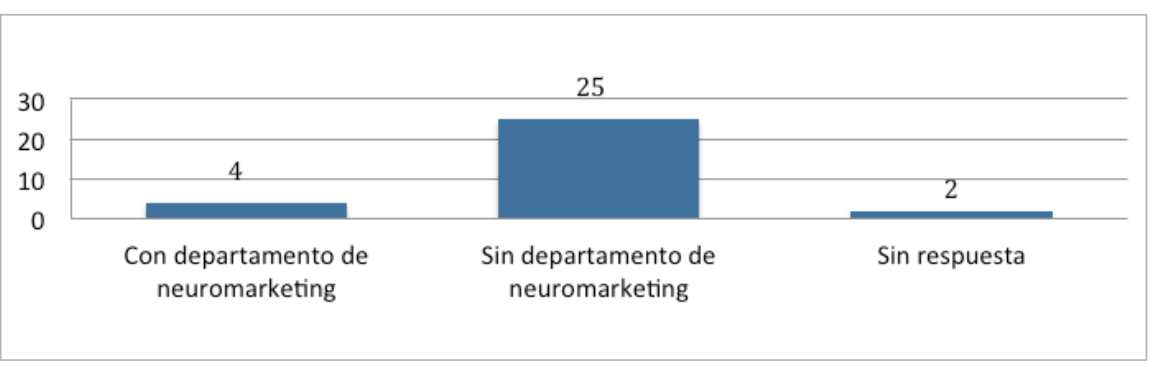

Figura 3. Relación de las agencias de publicidad y comunicación de Aeacp con el neuromarketing. instituto o centro que investiga en neuromarketing, y el $4 \%$ cuenta con algún grupo con investigación relacionada. Dos terceras partes de las universidades españolas no presentan ninguna línea de investigación directa o relacionada. No obtuvimos respuesta de 6 universidades (8\%).

Las universidades españolas que realizan investigación en neuromarketing o en temas relacionados son: $A$ Coruña, Autònoma de Barcelona, Autónoma de Madrid, Cádiz, Castilla La Mancha, Complutense de Madrid, Granada, La Rioja, Málaga, Miguel Hernández, Murcia, Oviedo, Pablo de Olavide, Politécnica de Cartagena, Pompeu Fabra, Rey Juan Carlos, Rovira i Virgili, València y Zaragoza.

La distribución por comunidades autónomas (tabla 1) muestra que la investigación se desarrolla principalmente en Andalucía, Madrid, la Comunidad Valenciana y Murcia.

Los resultados de las agencias de publicidad y comunicación (figura 3 y tabla 2) muestran que el 13\% cuenta con un departamento de neuromarketing u ofrecen este servicio a sus clientes. La mayoría (81\%) de agencias no cuentan con un departamento al respecto ni ofrecen este servicio a sus clientes. No obtuvimos respuesta de 2 de las 31 agencias contactadas.

Una quinta parte del sector publicitario profesional muestra interés real en neuromarketing

La encuesta realizada a las cuatro agencias que sí cuentan con departamento de neuromarketing u ofrecen dicho servicio a sus clientes (Gaps, Leo Burnett Iberia, McCann y VCCP) presenta los siguientes resultados:

- Dos de ellas lo subcontrata a empresas especializadas, una cuenta con un departamento de neuromarketing propio, y la otra no da una respuesta concreta.

- Tres ofrecen estudios de neuromarketing para optimizar los resultados y las necesidades que puedan tener sus clientes.

- Los principales clientes que solicitan este tipo de servicios pertenecen a los sectores del gran consumo (40\%) y del entretenimiento (20\%).

- Ofrecen estos servicios desde hace menos de dos años.

- La facturación correspondiente a los estudios de neuromarketing es inferior al $5 \%$ del total de las agencias.

La investigación en las empresas de investigación de mercados y opinión pública que forman Aneimo muestra que

el $37 \%$ cuenta con un departamento de neuromarketing u ofrece este servicio a sus clientes.

La encuesta a las seis empresas de Aneimo con departamento de neuromarketing o con este servicio en cartera (Análisis e Investigación, Block de Ideas S.L., Gfk Emer Market Research Solutions, Millward Brown Spain, Quota Research S.A. y TNS/Kantar) presenta los siguientes resultados:

- Cinco de ellas tienen departamento de neuromarketing propio, que está formado por un $45 \%$ de psicólogos y un $11 \%$ de ingenieros. El $44 \%$ restante de los departamentos están formados por sociólogos, publicistas y economistas principalmente. Llama la atención la ausencia de neuro-

Tabla 2. Las agencias de publicidad y comunicación de Aeacp con departamento o servicios de neuromarketing

\begin{tabular}{|c|c|c|}
\hline $\begin{array}{l}\text { Con departamento } \\
\text { de neuromarketing }\end{array}$ & $\begin{array}{c}\text { Sin departamento de neu- } \\
\text { romarketing }\end{array}$ & Sin respuesta \\
\hline Gaps & Arena 360 & Cheil Spain \\
\hline Leo Burnett Iberia & Arnold Madrid & Shackleton \\
\hline McCann & Bungalow25 & \\
\hline \multirow[t]{22}{*}{$V C C P$} & Contrapunto BBDO & \\
\hline & $D D B$ & \\
\hline & $D E C$ & \\
\hline & Del Campo Saatchi \& Saatchi & \\
\hline & Draftfcb Spain & \\
\hline & El Laboratorio & \\
\hline & Evora Marketing y Publicidad & \\
\hline & GMR Marketing & \\
\hline & Grey Group & \\
\hline & Havas Worldwide Spain & \\
\hline & Innocean Worldwide & \\
\hline & La Fórmula & \\
\hline & $J W T$ & \\
\hline & $\angle O L A$ & \\
\hline & Ogilvy \& Mather & \\
\hline & Publicis Comunicación & \\
\hline & Sra. Rushmore & \\
\hline & TBWA & \\
\hline & Tactis Europe & \\
\hline & Tapsa & \\
\hline & Tiempo/BBDO & \\
\hline & Zapping/M\&CSaatchi & \\
\hline
\end{tabular}




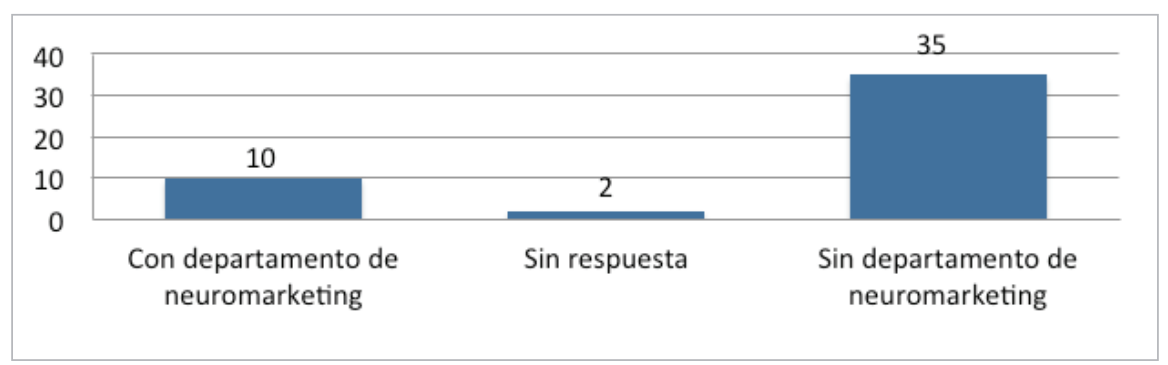

Figura 4. Resultados del sector profesional de la publicidad y el marketing en España.

desarrollo del neuromarketing en las empresas de investigación de mercados que en las agencias de publicidad y comunicación, puede que por la alta especialización necesaria en los estudios de neuromarketing.

Las cuestiones éticas sobre estos servicios preocupan mucho a la sociedad y cada vez implican más a las ciencias sociales. El hecho de

científicos en la recolección y análisis de los datos.

- Una subcontrata los servicios de neuromarketing a empresas especializadas.

- Los servicios de neuromarketing se presentan a los clientes como una herramienta capaz de optimizar los resultados y las necesidades de los mismos (33\%), como complementaria a las técnicas tradicionales de auto-reporte (34\%) y como una herramienta eficaz capaz de aumentar el número de ventas de los clientes (33\%).

- Los principales clientes pertenecen a los sectores del gran consumo (62\%) y automovilístico (13\%).

- La mayoría de las empresas ofrece este servicio desde hace 2 años o más.

- A lo largo del último año, el $40 \%$ ha realizado entre 10 y 30 estudios de neuromarketing, mientras que el $60 \%$ restante ha realizado menos de 10 .

- Este tipo de encargos suponen menos de un 5\% de la facturación total para 4 de las empresas. Una de ellas afirma obtener entre un 10 y un $20 \%$ de la facturación a través de servicios de neuromarketing.

Si tenemos en cuenta los resultados procedentes de las agencias de publicidad y de las empresas de investigación de mercados de manera conjunta (figura 4), obtenemos que la quinta parte del sector profesional de la publicidad y el marketing en España (un 21,3\%) cuenta con un departamento de neuromarketing $u$ ofrece dicho servicio en su cartera. La mayor parte del sector profesional, un 74,5\%, no trabaja en neuromarketing.

\section{Discusión y conclusiones}

El neuromarketing en España presenta un interés creciente en la investigación y en el ámbito profesional. Una cuarta parte de las universidades españolas están realizando investigación sobre neuromarketing. Teniendo en cuenta la realidad actual de la investigación en España, el neuromarketing es una disciplina emergente al igual que en otros países. Sin embargo, si atendemos a criterios de publicación científica internacional, observamos que en los últimos años (de 2004 a octubre de 2013) sólo 3 publicaciones realizadas por grupos españoles están incluidas en la base de datos WoS de Thomson Reuters. Buena parte de las investigaciones se hacen al margen de la investigación neurocientífica estableci$\mathrm{da}$, aunque mediante equipos multidisciplinares, donde no siempre hay neurocientíficos.

Por su parte, una quinta parte del sector publicitario profesional muestra interés real en neuromarketing, bien con un departamento propio, bien ofreciendo en su cartera dichos servicios contratados a terceros. Se produce un mayor que no exista una normativa propia de neuromarketing que obligue a toda investigación profesional realizada en este área a tener el aval de un comité de ética, provoca que buena parte de la investigación profesional llevada a cabo en esta línea carezca de registros públicos y transparentes. Por ello, sería interesante continuar esta línea de trabajo para averiguar cómo regula la actual legislación la investigación neurocientífica en España y qué repercusiones tiene carecer del aval científico de un comité de ética en la investigación en neuromarketing. Es posible que estemos ante un gran avance del conocimiento que se está llevando a cabo en el ámbito profesional y que, por intereses económicos de los clientes (marcas o agencias) no se acaba haciendo público entre la sociedad, en general, y entre todos los profesionales y teóricos de la comunicación, en particular.

Es posible que estemos ante un gran avance del conocimiento que se lleva a cabo en el entorno profesional y que por intereses económicos no se hace público

Por otro lado, este trabajo abre el camino para continuar investigando las técnicas neurocientíficas (electroencefalografía, resonancia magnética funcional, tomografía por emisión de positrones, etc.) que actualmente están teniendo una mayor incidencia en el área de la publicidad y el marketing en España. Igualmente entendemos que es necesario mantener esta línea para conocer la eficacia de cada una de estas técnicas en el área de la comunicación y así ir acotando la utilidad, o no, del incipiente campo de estudio del neuromarketing.

\section{Bibliografía}

Andreu-Sánchez, Celia; Martín-Pascual, Miguel-Ángel (2014). "Selling the brain: Representation of neuroscience in advertising". En: Grabowski, Michael (ed.) Media on the brain. En prensa.

Ariely, Dan; Berns, Gregory S. (2010). "Neuromarketing: the hope and hype of neuroimaging in business". Nature reviews. Neuroscience, April, v. 11, n. 4, pp. 284-292. http://www.ncbi.nlm.nih.gov/pmc/articles/PMC2875927 http://dx.doi.org/10.1038/nrn2795

Asociación de Empresas de Investigación de Mercados y Opinión. Asociados y sus servicios.

http://www.aneimo.com/asociados.php 
Asociación Española de Agencias de Comunicación Publicitaria. Agencias asociadas.

http://www.agenciasaeacp.es/agencias/agencias_asociadas.asp

Braidot, Néstor (2007). Neuromarketing ¿Por qué tus clientes se acuestan con otro si dicen que les gustas tú? Barcelona: Editorial Gestión 2000. ISBN: 9788498750447

Eaton, Margaret L.; Illes, Judy (2007). “Commercializing cognitive neurotechnology - the ethical terrain". Nature biotechnology, April, v. 25, n.4, pp. 393-397.

http://dx.doi.org/10.1038/nbt0407-393

Hubert, Mirja; Kenning, Peter (2008). "A current overview of consumer neuro-science". Journal of consumer behaviour, July, v. 7, n. 4-5, pp. 272-292.

http://dx.doi.org/10.1002/cb.251

Javor, Andrija; Koller, Monika; Lee, Nick; Chamberlain, Laura; Ransmayr, Gerhard (2013). "Neuromarketing and consumer neuroscience: contributions to neurology". BMC neurology, February, v. 13, pp. 1-12.

http://dx.doi.org/10.1186/1471-2377-13-13

Khushaba, Rami N.; Wise, Chelsea; Kodagoda, Sarath; Louviere, Jordan; Kahn, Barbara E.; Townsend, Claudia (2013). "Consumer neuroscience: Assessing the brain response to marketing stimuli using electroencephalogram (EEG) and eye tracking". Expert systems with applications, July, v. 40, n. 9, pp. 3803-3812.

http://www.cs.tau.ac.il/ nin/Courses/Seminar14a/ Neuromarketing.pdf

http://dx.doi.org/10.1016/j.eswa.2012.12.095

Lee, Nick; Broderick, Amanda J.; Chamberlain, Laura (2007). "What is 'neuromarketing'? A discussion and agenda for future research". International journal of psychophysiology, February, v. 63, n. 2, pp. 199-204.

http://dx.doi.org/10.1016/j.ijpsycho.2006.03.007

McClure, Samuel M.; Li, Jian; Tomlin, Damon; Cypert, Kim S.; Montague, Latané M.; Montague, P. Read (2004). “Neural correlates of behavioral preference for culturally familiar drinks". Neuron, October, v. 44, n. 2, pp. 379-387.

http://www.commercialalert.org/neuromarketingcokepepsi.pdf http://dx.doi.org/10.1016/j.neuron.2004.09.019

Murphy, Emily R.; Illes, Judy; Reiner, Peter B. (2008). “Neuroethics of neuromarketing". Journal of consumer behaviour, July, v. 7, n. 4-5, pp. 293-302.

http://dx.doi.org/10.1002/cb.252

Plassmann, Hilke; Ambler, Tim; Braeutigam, Sven; Kenning, Peter (2007). "What can advertisers learn from neuroscience?" International journal of advertising, v. 26, n. 2, pp. 151-175.

http://psycnet.apa.org/psycinfo/2007-08623-001

Plassmann, Hilke; Zoëga, Thomas; Milosavljevic, Milica (2012). "Branding the brain: A critical review and outlook". Journal of consumer psychology, January, v. 22, pp. 18-36. http://dx.doi.org/10.1016/j.jcps.2011.11.010
Plöchl, Michael; Ossandón, José P.; König, Peter (2012). "Combining EEG and eye tracking: identification, characterization, and correction of eye movement artifacts in electroencephalographic data". Frontiers in human neuroscience, October, v. 6, n. 278, pp. 1-23.

http://dx.doi.org/10.3389/fnhum.2012.00278

Padreep, A. K. (2010). The buying brain: secrets for selling to the subconscious mind. New Jersey: John Wiley \& Sons. ISBN: 9780470601778

QS Quacquarelli Symonds Limited. QS World University Ranking 2013.

http://www.topuniversities.com/university-rankings/worlduniversity-rankings $/ 2013$ \#sorting $=$ rank+region $=+$ country $=+$ faculty $=+$ stars $=$ false + search $=$

Randall, Kevin (2009). Neuromarketing hope and hype: 5 brands conducting brain research. Fast Company.

http://www.fastcompany.com/1357239/neuromarketinghope-and-hype-5-brands-conducting-brain-research

Santaló, Josep (2013). Entrevista personal.

Smidts, Ale (2002). Kijken in het brein: Over de mogelijkheden van neuromarketing. Rotterdam: Erasmus Research Institute of Management - Inaugural lectures. ISBN: 978 9058920362

Touhami, Zineb-Ouazzani; Benlafkih, Larbi; Jiddane, Mohamed; Cherrah, Yahya; El Malki, Hadj-Omar; Benomar, Ali (2011). "Neuromarketing: Where marketing and neuroscience meet". African journal of business management, March, v. 5, n. 5, pp. 1528-1532.

http://dx.doi.org/10.5897/AJBM10.729

TSL Education Ltd. The times higher education 100 under 50 universities 2013.

http://www.timeshighereducation.co.uk/world-universityrankings/2013/one-hundred-under-fifty

Venkatraman, Vinod; Clithero, John A.; Fitzsimons, Gavan J.; Huettel, Scott A. (2012). "New scanner data for brand marketers: How neuroscience can help better understand differences in brand preferences". Journal of consumer psychology, v. 22, n. 1, pp. 143-153.

http://www.hss.caltech.edu/ clithero/Venkatraman_ JCP_12.pdf

http://dx.doi.org/10.1016/j.jcps.2011.11.008

Weisberg, Deena-Skolnick; C. Keil, Frank; Goodstein, Joshua; Rawson, Elizabeth; Gray, Jeremy R. (2008). "The seductive allure of neuroscience explanations". Journal of cognitive neuroscience, March, v. 20, n. 3, pp. 470-477.

http://www.ncbi.nlm.nih.gov/pmc/articles/PMC2778755 http://dx.doi.org/10.1162/jocn.2008.20040

Wilson, R. Mark; Gaines, Jeannie; Hill, Ronald-Paul (2008). "Neuromarketing and consumer free will". The journal of consumer affairs, v. 42, n. 3, pp. 389-410.

http://class.classmatandread.net/Physio/neurofreewill.pdf http://dx.doi.org/10.1111/j.1745-6606.2008.00114. 